Edición Extraordinaria. p.p. 389 - 398

Memorias del VIII Encuentro Nacional de Experiencias en Enseñanza de la Biología y la Educación Ambiental. III Congreso Nacional de Investigación en Enseñanza de la Biología.

\title{
Pequeño planeta sostenible - una práctica interdisciplinaria en Educación Ambiental en la Educación Superior
}

Sustainable small planet - an interdisciplinary practice in Environmental Education in Higher Education

Elizabeth Aparecida Assis Brandão Danhão 1 Simone Oliveira Amadeu ${ }^{2}$

Rita de Cássia Frenedozo ${ }^{3}$

Vitor Viana dos Santos ${ }^{4}$

\section{Resumo}

Este trabajo se sugiere la realización de una actividad práctica en la educación ambiental para sensibilizar y aclarar la sostenibilidad en la escuela. Esta actividad se llevó a cabo un taller para 70 estudiantes de una universidad privada ubicada en el estado de São Paulo-SP, Brasil, para un evento titulado "Semana de Estudio", celebrado en octubre de 2013. El diferencial esta actividad práctica aplica el taller es el enfoque interdisciplinario, que busca replicabilidade en el aula, ya que los estudiantes de educación superior pueden transmitir los conceptos y prácticas desarrolladas por los diferentes niveles educativos de manera multi o interdisciplinaria. La práctica sugiere mejorar la educación ambiental a través interdisciplinario mediante la reutilización de vidrio y / o plástico ollas para crear un sistema en el que se pueden observar: los ecosistemas, el ciclo hidrológico, el desarrollo de los seres vivos él y el equilibrio perfecto esta introducido sin la acción los seres humanos, que simulan un equilibrio planeta Mini.

Palavras chave: la enseñanza de la práctica; educación ambiental; sostenibilidad

\section{Abstract}

In this paper we suggest the realization of a practical activity in environmental education to raise awareness and clarify sustainability in school. This activity was

\footnotetext{
${ }^{1}$ Mestranda em Ensino de Ciências, Universidade Cruzeiro do Sul-SP, Bolsista CAPES,

${ }^{2}$ Mestra em Ensino de Ciências, Universidade Cruzeiro do Sul-SP.

${ }^{3}$ Professora titular da Pós graduação em Ensino de Ciências, Universidade Cruzeiro do Sul, São Paulo, SP

${ }^{4}$ Estudante de Graduação em Ciências Biológicas pelo Centro Universitário Nossa Senhora do Patrocínio, Itu, SP. 
Edición Extraordinaria. p.p. 389 - 398

Memorias del VIII Encuentro Nacional de Experiencias en Enseñanza de la Biología y la Educación Ambiental. III Congreso Nacional de Investigación en Enseñanza de la Biología.

carried out by a workshop for 70 students college students of a private university located in the state of São Paulo-SP, Brazil during an event titled "Study Week" held in October 2013. The differential this practice activity applied the workshop is the interdisciplinary approach, which seeks to replicability in the classroom, as students of higher education can pass on the concepts and practices developed for different educational levels of multi- or interdisciplinary way. The practice suggests enhance environmental education through interdisciplinary by reusing glass and / or plastic pots to create a system where they can be observed: ecosystems, the hydrological cycle, the development of living beings introduced him and the perfect balance this without the action humans, simulating a mini planet balance.

Key words: teaching practice; environmental education; sustainability 
Bio - grafia. Escritos sobre la Biología y su Enseñanza. ISSN 2027

Edición Extraordinaria. p.p. 389 - 398

Memorias del VIII Encuentro Nacional de Experiencias en Enseñanza de la Biología y la Educación Ambiental. III Congreso Nacional de Investigación en Enseñanza de la Biología.

\section{Pequeño planeta sostenible - una práctica interdisciplinaria en Educación Ambiental en la Educación Superior}

\section{Introducción}

Las cuestiones ambientales están ahora en pruebas debido a las constantes necesidades de preservación y equilibrio ambiental. La educación ambiental juega un papel importante en la construcción interdisciplinaria valores sociales, conocimientos y habilidades, actitudes y competencias destinadas a preservar el medio ambiente, que es un uso común (Rivelli, 2005). A través de la educación ambiental que podemos ilustrar, orientar y educar a la gente sobre la importancia de la preservación, la conservación y el cuidado de la continuidad de la vida en el planeta. Para ampliar la conciencia sobre la importancia de preservar proponer la producción de una práctica pedagógica llamada " pequeño planeta " sostenible, que consiste en una dinámica interdisciplinaria aplicables en el aula para ilustrar la sostenibilidad y la preservación del planeta.

\section{Un poco sobre Educación Ambiental}

Educación ambiental surgió de la necesidad de educar a la gente sobre la importancia del conocimiento, conservación de la naturaleza, los recursos naturales y las soluciones a los problemas ambientales. Este término se produjo en 1972, la primera Conferencia Mundial para el Medio Ambiente Humano en Estocolmo (Suecia) en poder del (Naciones Unidas) de las Naciones Unidas. No fue sino hasta 1975 que la UNESCO (UNESCO) ha tomado la responsabilidad de organizar y discutir la educación ambiental de carácter regional e internacional. Desde que la educación ambiental se ha convertido en una herramienta para ayudar en las técnicas de aprendizaje.

Según Sauvé (2005), es una dimensión esencial de la educación básica centrada en una esfera de interacción que se encuentra en la base del desarrollo personal y social: la euforia con el medio ambiente en que vivimos, con este "vivir en casa "compartido. Más de una educación "sobre, por, sobre, por o en nombre de" el medio ambiente, el objeto de la educación es de hecho fundamentalmente nuestra relación con el medio ambiente (Sauvé, 
Bio - grafia. Escritos sobre la Biología y su Enseñanza. ISSN 2027

Edición Extraordinaria. p.p. 389 - 398

Memorias del VIII Encuentro Nacional de Experiencias en Enseñanza de la Biología y la Educación Ambiental. III Congreso Nacional de Investigación en Enseñanza de la Biología.

2005, p.1).

La educación ambiental es muy complejo porque abarca diferentes aspectos, es un tema interdisciplinario y esencial para la supervivencia del planeta. Entre ellos podemos relacionar: el medio ambiente de acuerdo con las relaciones interpersonales entre los seres humanos con la naturaleza en el sentido de la conservación; el medio ambiente.

\section{Teórica Fundación}

Para Vygotsky (1994), "los elementos mediadores en la relación entre el hombre y el mundo se hacen en las relaciones entre los hombres...". Estos elementos consisten en herramientas, signos y todos los elementos de la naturaleza humana riegan significado cultural, constituidas por las relaciones entre los seres humanos. Los elementos mencionados por Vigotsky consisten en herramientas, signos y elementos de la naturaleza". Para él la naturaleza y sus acciones colectivas también puede influir en la formación del ciudadano e interferir en el proceso de enseñanza y aprendizaje, tanto en la visión social, cultural y ambiental.

En el punto de vista educativo que defiende la teoría de que el maestro interviene en el proceso de aprendizaje mediante la participación en el balance de la mediación entre la razón y la emoción, constituyendo un individuo crítico y social. La educación social juega un papel fundamental en el desarrollo y el aprendizaje individual que utiliza señales de interpretar esta relación con el entorno en el que aparece. Pensando en ello es que apoyamos la actividad, ese puente entre la teoría y la práctica de facilitar el proceso de enseñanza y aprendizaje.

Los estudiantes hacen sus pensamientos y recuerdos a través de intercambios de experiencias y el diálogo. Este entrenamiento mental es voluntaria y se construye durante toda la vida. "El significado de todo lo que está en las personas y las cosas, pero no puede ser las cosas si no son las personas, por lo tanto, el papel de las emociones" (Vygotsky, 1994).

La actividad práctica provoca reacciones a los estímulos de diferentes emociones en los estudiantes, ya que fomenta el uso de diferentes direcciones al mismo tiempo, lo que facilita el aprendizaje y la memorización. 
Bio - grafia. Escritos sobre la Biología y su Enseñanza. ISSN 2027

Edición Extraordinaria. p.p. 389 - 398

Memorias del VIII Encuentro Nacional de Experiencias en Enseñanza de la Biología y la Educación Ambiental. III Congreso Nacional de Investigación en Enseñanza de la Biología.

\section{La interdisciplinariedad}

La interdisciplinariedad es también una importante dificultad que enfrentan los maestros que no ven con claridad y facilidad, cómo aplicar esta técnica en la vida escolar cotidiana. Conceptualizar la interdisciplinariedad es un gran desafío, así como que sea práctico en la vida escolar cotidiana. La cuestión de las definiciones también se extiende a sus respectivos clientes potenciales, como multidisciplinario, transdisciplinario, multidisciplinario, entre otros.

Nuestro objetivo no es buscar la justificación epistemológica de todos los ajustes y variaciones que se encuentran en disciplinaria, pero resaltarlos en cuestiones pedagógicas y guiar a los estudiantes que participan en la actividad práctica propuesta por su importancia como una ayuda en el proceso de enseñanza y aprendizaje.

Según Pombo (2003) es más fácil de buscar definiciones en la raíz de la palabra interdisciplinario que ponen su definición real. Sobre la base de esta hipótesis, para definir disciplinaria, que se originó a partir de la palabra disciplina puede definir, ella tres significados principales:

"La disciplina como una rama del conocimiento: las matemáticas, la física, la biología, la sociología o la psicología son las disciplinas, las ramas del conocimiento o, más bien, algunas de estas ramas. Luego tenemos las subdisciplinas y así sucesivamente. La disciplina como componente curricular: Historia, Ciencias Naturales, Cristalografía, Química Inorgánica, etc. Por supuesto, en gran medida, muchas de las materias del currículo se cortan en científica acompañar a su surgimiento, su desarrollo, aunque, como sabemos, siempre con el tiempo y los efectos de cambio inexorables GAL. Por último, la disciplina como un conjunto de reglas o leyes que regulan una determinada actividad o conducta de un grupo en particular: la disciplina militar, la disciplina fabricante de automóviles o la escuela disciplina, etc. (Pombo, 2003, pp.12-13)."

Según ha Lenoir (1998), las opciones epistemológicas para la interdisciplinariedad de la escuela se han caracterizado por el establecimiento de conexiones entre dos o más disciplinas (enfoque relacional), o los conceptos o amplio espectro de temas de estudio, destacando la sustitución del conocimiento dividido en disciplinas por una unidad de conocimiento o de un sujeto (enfoque radical). 
Bio - grafia. Escritos sobre la Biología y su Enseñanza. ISSN 2027

Edición Extraordinaria. p.p. 389 - 398

Memorias del VIII Encuentro Nacional de Experiencias en Enseñanza de la Biología y la Educación Ambiental. III Congreso Nacional de Investigación en Enseñanza de la Biología.

En las Directrices Curriculares Nacionales para Escuelas Secundarias (DCNEM), "la interdisciplinariedad debe entenderse desde un enfoque relacional" (BRASIL, 2002, p. 36).

Incluso con las definiciones y los diferentes orígenes puede darse cuenta de que la interdisciplinariedad es un paradigma, pero es crucial para fortalecer las relaciones de disciplina y contribuir a la ejecución de proyectos. Ya sea inter, multi, multi o transdisciplinario.

Según Fazenda (2002, p.66), "la difuminación de la interdisciplinariedad todavía se levantan las ideas erróneas sobre el concepto de disciplina." La controversia sobre disciplina e interdisciplinario permite a una dificultad en la que la acción se convierte en el punto de convergencia entre el hacer y el pensamiento interdisciplinario. Tenemos que relacionar $y$ iterando las disciplinas que sería el sello distintivo de las relaciones interdisciplinarias.

\section{Los objetivos de esta actividad}

En las actividades prácticas, el profesor puede ilustrar una conferencia que busca la comprensión de sus alumnos, aunque inconsciente, demostrar que el estudiante es una parte integral de la naturaleza y que también depende del equilibrio necesario para que se produzca el ciclo natural de todos. Esta toma de conciencia es esencial para la educación ambiental se lleve a cabo y el resultado final el equilibrio del medio ambiente y la preservación del planeta.

A través de esta práctica interdisciplinar de la educación ambiental, queremos que el estudiante de educación superior, el futuro (a) maestro (a), puede aprender y replicar esta actividad práctica en el aula. De esta manera contribuir al desarrollo de una conciencia y actitudes de preservación del medio ambiente. Con esta práctica los estudiantes pueden observar que el planeta tiene un equilibrio natural. Eso sin la interferencia agresiva del hombre, se establece el ciclo de vida y la Tierra se convierte en armonía en sus ecosistemas.

Muchas de las actividades interdisciplinarias se pueden desarrollar con esta práctica, que facilita el conocimiento de la importancia de la preservación, la conservación y la conciencia ambiental. 
Bio - grafia. Escritos sobre la Biología y su Enseñanza. ISSN 2027

Edición Extraordinaria. p.p. 389 - 398

Memorias del VIII Encuentro Nacional de Experiencias en Enseñanza de la Biología y la Educación Ambiental. III Congreso Nacional de Investigación en Enseñanza de la Biología.

\section{Metodología}

Los sujetos de esta investigación fueron 70 estudiantes de educación superior de los cursos de Ciencias Biológicas (licenciatura y licenciados), Letras y Pedagogía de una universidad privada en el estado de São Paulo, Brasil. Se celebraron dos talleres durante la semana de estudio el 17 de octubre de 2013, que dura alrededor de una hora y media en el laboratorio / Biología Botánica. Una presentación de diapositivas sobre la importancia de la educación ambiental, el fomento de su práctica en el aula, la información y orientación sobre la actividad práctica se presentaron inicialmente a los estudiantes para que puedan replicarlos. Después de las orientaciones teóricas estudiantes realizan la práctica de la construcción sostenible de los planetas y se llevaron a casa para hacer observaciones diarias en sus respectivos productos.

Para desarrollar esta actividad práctica utilizado las siguientes herramientas metodológicas: la observación directa y la recogida de testimonios de los involucrados.

Como futuros profesionales de la educación, se nos dijo que esta actividad práctica se recomienda para los diferentes grupos de edad, dependiendo del alcance de las cuestiones y problemas multi o interdisciplinarios que se abordarán en el proyecto. Es una actividad sencilla que permite una amplia gama de temas relacionados y el maestro debe dirigirlos para determinar la cuestión a destacar con la actividad. Algunas sugerencias para diferentes grupos de edad:

- En la educación infantil - sólo puede ocurrir la observación diaria de los fenómenos que ocurren en el pequeño planeta;

- En la educación primaria I y II - el estudiante puede participar activamente, a partir de materiales que obtienen y en su montaje y observación;

- En la escuela secundaria - Además de los consejos anteriores estudiantes pueden relacionar los distintos temas que se pueden abordar con esta práctica, tales como los fenómenos meteorológicos, los ecosistemas, entre otros;

- En Educación Superior - todos los temas disciplinarios e interdisciplinarios pueden profundizarse, haciendo que la actividad compleja e interactiva. 
Bio - grafia. Escritos sobre la Biología y su Enseñanza. ISSN 2027

Edición Extraordinaria. p.p. 389 - 398

Memorias del VIII Encuentro Nacional de Experiencias en Enseñanza de la Biología y la Educación Ambiental. III Congreso Nacional de Investigación en Enseñanza de la Biología.

\section{Materiales y Procedimientos}

Para el taller se les hizo a los estudiantes a tomar lo lava y cristal o maceta de plástico esterilizado. Hacemos hincapié en que es esencial que los alumnos comprendan que estos materiales se reutilizan y se pueden encontrar en la vida cotidiana, sin costo alguno, y es a través de este que ilustran la facilidad y la importancia de la reutilización para la vida de cada uno.

Para esta actividad se llevó: vidrios transparentes plásticos usados, mas lavados e higienizados:

- grava;

- Tierra;

- Agua;

- Película de plástico;

- planta no sensibles cambie el agua de las hojas;

Después de la reunión de todos los materiales a los estudiantes se les dijo acerca de la importancia de la limpieza de vidrios o macetas para que no se produzcan interferencias en el ecosistema. La grava, se colocaron los cambios de plantas de la tierra y el agua. Después de regar, se pidió a los estudiantes para cubrir el recipiente con papel plástico de modo que simula la capa de ozono.

\section{Desarrollo}

El desarrollo de la actividad práctica era rápido y muy interactiva. Durante el montaje del sistema de los estudiantes "pequeño planeta" estaban recibiendo orientación sobre las capas del suelo, sobre los seres en los ciclos biogeoquímicos de estar, en la capa de ozono, entre otras informaciones.

Después de montar el "pequeño planeta", los estudiantes fueron instruidos en cómo estimular las observaciones diarias y la posible aplicabilidad en diferentes disciplinas y programas de estudio. Los estudiantes se les dijo acerca del análisis del entorno biótico y abiótico, la relación de dependencia y la armonía entre ellos y la naturaleza de los ciclos biogeoquímicos que son visibles en esta práctica. 
Bio - grafia. Escritos sobre la Biología y su Enseñanza. ISSN 2027

Edición Extraordinaria. p.p. 389 - 398

Memorias del VIII Encuentro Nacional de Experiencias en Enseñanza de la Biología y la Educación Ambiental. III Congreso Nacional de Investigación en Enseñanza de la Biología.

Otro contenido interdisciplinario directriz recibir referenciaba que se puede aplicar al conjunto de este sistema. Hacemos hincapié en que hay muchos variada y de acuerdo con la serie o el desarrollo de proyectos de contenido de nivel que los estudiantes pueden realizar muchas otras observaciones que pueden ser objetivo y documentadas. Para esta práctica no existe una regla o desarrollo aplicable porque es flexible, multidisciplinaria y puede encajar en cualquier actividad y / o teoría. Esta actividad se puede aplicar antes o después de la teoría.

Al final del taller, los estudiantes tomaron sus "planetinhas" producidos en su propia casa, con el fin de que pudieran experimentar, analizar y observar el proceso de desarrollo de sistemas en vista de los estudiantes y no docentes.

Siendo un taller aplicado en un evento específico, no seguir y registrar los resultados individuales propuestas a los estudiantes en esta actividad interdisciplinaria. Las observaciones se limitan a la fecha de aplicación de esta actividad, por lo que los resultados de esta experiencia práctica interdisciplinar en la educación ambiental se limita a observaciones durante la interactividad del taller y los comentarios de los estudiantes durante el desarrollo y en el final de la actividad. Al final del taller, los estudiantes demostraron actividad como práctica a través de las siguientes afirmaciones:

"Montaje muy interesante de que" pequeño planeta "porque podemos a través de esta actividad realice una práctica experimental que estimula la conciencia ambiental entre los estudiantes de manera interdisciplinaria" - informe en su totalidad de un estudiante de Ciencias Biológicas del cuarto semestre.

Una zona de lectura del alumno, Códigos y sus Tecnologías comentó: "Me encantó! Estimulado mi creatividad en las actividades prácticas de programación en mis clases futuras".

\section{Consideraciones finales}

Las actividades prácticas propuestas, educación ambiental interdisciplinaria en la educación superior, con objetivos de formación de multiplicadores capaces de replicar los conocimientos y prácticas es prometedor y necesario porque a través de estos nuevos profesionales podemos ampliar las actitudes y capacitar a más personas protagonistas, 
Bio - grafia. Escritos sobre la Biología y su Enseñanza. ISSN 2027

Edición Extraordinaria. p.p. 389 - 398

Memorias del VIII Encuentro Nacional de Experiencias en Enseñanza de la Biología y la Educación Ambiental. III Congreso Nacional de Investigación en Enseñanza de la Biología.

autónomos y conscientes de su papel y la importancia en la sociedad en la que aparece. A través de este taller para construir un "planeta sostenible" buscar la replicabilidad de la sostenibilidad natural cuando el hombre está ausente de la agresión y la degradación.

También queremos ilustrar a través de esta actividad para nuestros estudiantes que se pueda llevar a cabo acciones simples que ayudan en la preservación y conservación del planeta.

\section{Referencias}

BRASIL. Parâmetros Curriculares Nacionais, e Ensino Médio. "Orientações Educacionais Complementares aos Parâmetros Curriculares Nacionais." Ciências da Natureza, Matemática e suas Tecnologias, 2002.

FAZENDA, Ivani Catarina Arantes. Integração e interdisciplinaridade no ensino brasileiro. Edições Loyola, 2002.

LENOIR, Y. Didática e interdisciplinaridade: uma complementaridade necessária e incontornável. In: Didática e interdisciplinaridade. FAZENDA, I. C. A. (org.). Campinas: Papirus, 1998, p. 45-76.

POMBO, Olga. Epistemologia da interdisciplinaridade. Seminário Internacional Interdisciplinaridade, Humanismo, Universidade. (Porto, 2003). Porto: Universidade do Porto, p. 1-18, 2003.

RIVELLI, E. A. L. "Evolução da Legislação Ambiental no Brasil: Políticas de Meio Ambiente, Educação Ambiental e Desenvolvimento Urbano in PHILIPPI, Jr; PELICIONI, MCF Educação Ambiental e Sustentabilidade." Barueri, SP (2005).

SAUVÉ, Lucie. "Educação ambiental e desenvolvimento sustentável: uma análise complexa." Revista de Educação Pública 6.10 (1997): 72-103.

VYGOTSKY, Liev S. A formação social da mente: o desenvolvimento dos processos psicológicos superiores. 5 ed. São Paulo: Martins Fontes, 1994. 